\title{
Effects of large-scale AGN feedback in local galaxies
}

\author{
Stanislav Shabala ${ }^{1}$, Sugata Kaviraj ${ }^{2}$ and Joseph Silk ${ }^{2}$ \\ ${ }^{1}$ School of Mathematics \& Physics, Univ. of Tasmania, Private Bag 37, Hobart 7001, Australia \\ email: Stanislav.Shabala@utas.edu.au \\ ${ }^{2}$ Department of Physics, University of Oxford, Keble Road, Oxford, OX1 3RH, UK
}

\begin{abstract}
The effects of active galactic nucleus (AGN) feedback on group and cluster galaxies are investigated. We examine the colors of non-AGN hosts (i.e. satellite galaxies) by comparing galaxies overrun by radio AGN with similar galaxies located outside the radio AGN contours. We find that powerful Fanaroff-Riley type II (edge-brightened) radio AGN truncate star formation in the galaxies overrun by AGN-driven bow shocks. On the other hand, the ubiquitous FanaroffRiley type I (core-dominated) AGN do not affect neighboring galaxies. This result shows that, despite their rarity, feedback from powerful radio AGN is an important factor in the evolution of group/cluster galaxies.
\end{abstract}

Keywords. galaxies: active — galaxies: evolution — galaxies: photometry

We cross-matched group/cluster radio AGN from the NVSS survey with optical photometry from SDSS. Figure 1 shows distributions of colors for galaxies overrun by radio sources, compared with galaxies projected to lie outside the radio source contours. There is a clear dichotomy between FR-I and FR-II radio sources. Galaxies overrun by the edge-brightened FR-II sources show a deficit of blue colors (corresponding to less recent star formation) compared to the control sample. No such difference exists for FR-Is. The lines correspond to models in which FR-II driven bow shocks have truncated star formation either 10 or 100 Myrs ago. The median AGN age in our sample is 43 Myrs.

The models show that truncation of star formation by the AGN can explain the observed color transition in galaxies around FR-II radio sources. In other words, starting with the "unaffected" colors distribution, AGN feedback from powerful radio sources causes the observed reddening of satellite galaxy colors. Thus, a single AGN can affect the evolution of multiple galaxies.
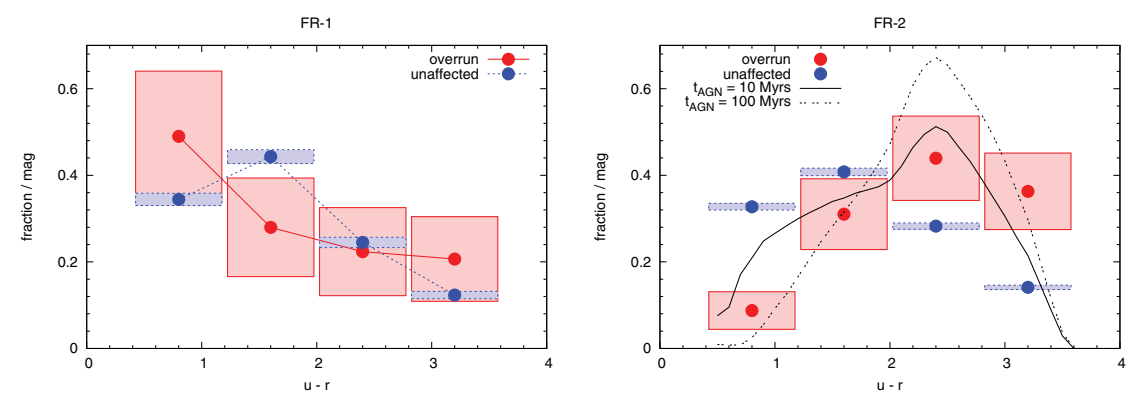

Figure 1. Mass-corrected $u-r$ colors around FR-I (left) and FR-II (right) AGN.

\section{Reference}

Shabala, S. S., Kaviraj, S., \& Silk, J. 2011, MNRAS 413, 2815 